\title{
Neighbor Discovery in Multichannel Wireless Clique Networks: An Epidemic Approach
}

\author{
Antonio Gonga, Themistoklis Charalambous, Mikael Johansson \\ School of Electrical Engineering - Royal Institute of Technology \\ SE-100 44 STOCKHOLM-Sweden \\ \{gonga, themisc, mikaelj\}@kth.se
}

\begin{abstract}
We investigate the problem of neighbor discovery in multichannel wireless ad hoc and sensor networks with epidemic information dissemination. Previous works have considered neighbor discovery in a single channel where at most one node can be discovered per time instant. To reduce the effect of collisions observed in single channel solutions, we formulate models for multichannel neighbor discovery and allow for epidemic dissemination of information. As a result, nodes can discover all their neighbors faster, either directly or indirectly by hopping between orthogonal channels and exploring the neighbors in each of them. We show analytically, by simulations, and by experimental evaluations that the expected neighbor discovery time is reduced considerably compared to singlechannel neighbor discovery solutions.
\end{abstract}

Index Terms-Neighbor discovery, sensor networks, epidemic algorithms, multichannel

\section{INTRODUCTION}

Emerging network applications require short range and/or local communications in distributed and ad-hoc manner. At the same time, it is well known that the quality of the communication channels in wireless networks can evolve quickly [1]. Hence, continuous neighbor discovery is becoming critical for keeping the neighborhood topology updated, and also to find routes/pairs to communicate with. The existence of many wireless devices operating on the same channel is a source of interference and collisions. Therefore, it is a critical for an efficient neighbor discovery algorithm to limit the collision probability to reduce the expected time required for all nodes to discover all their respective neighbors.

Neighbor discovery can be distinguished into two main categories: randomized [2] and deterministic [3]. The first category includes algorithms where each node takes randomly decides whether to transmit or listen, whereas in the second category (deterministic algorithms) there is a pre-allocated schedule. In this work, we concentrate on randomized algorithms that can be easily implemented in a distributed fashion.

In dense deployments, single-channel neighbor discovery is inefficient, because, the successful transmission probability decreases with the increase of the network size. The number of collisions can be reduced by multi-channel communications [4], offered by emerging standards such as IEEE 802.15.4e [5]. On the other hand, using multiple channels for neighbor discovery on low-power half-duplex transceivers rises many new challenges. For example, choosing an arbitrary large number of channels for a small network size it becomes harder for nodes to find each other on the same channel, since nodes can only listen to one channel at a time. While a small number of channels for a larger network may not reduce effectively the number of collisions. However, when nodes join or leave the network, fast detection and information dissemination is required, since the arrival or failure of a node causes many links to collapse. To compensate for this effect, we introduce an epidemic neighbor information dissemination component. Epidemic algorithms have been used in peer-topeer systems and database replication in wired networks [6], [7], as well as for gossiping in radio networks [8]. Although multichannel communications have been employed before in neighbor discovery (see, for example, [9], [10]), to the best of our knowledge, epidemic information dissemination was never integrated in neighbor discovery. This work aims to fill in this gap.

In this paper, we develop distributed randomized algorithms for multichannel neighbor discovery. Specifically, we focus on low-power half-duplex transceivers, dominantly used in sensor networks. Our contributions are the following:

(i) We propose a multichannel neighbor discovery algorithm with epidemic information dissemination that is shown to reduce the neighbor discovery time compared to the single channel, single packet reception model.

(ii) We explore the impact of the number of channels on the expected neighbor discovery time with respect to the total number of nodes in the network. It is shown that, apart from networks with a very few nodes, increasing the number of channels improves the neighbor discovery time when the number of nodes increases.

(iii) We show via simulations and experimental evaluation, the effectiveness of our proposed protocol. The suggested protocol could be used to extend, for example, the beaconing mechanism in existing standard protocols, such as the IEEE 802.15.4e.

The remainder of this paper is structured as follows. Section II, presents the network model and the problem formulation. In Section III, we extend the single channel case to the multichannel neighbor discovery problem with epidemic information dissemination. In Section V, the performance of the proposed protocol is evaluated and compared with existing state-of-theart. Finally, in Section VI, we provide our final remarks and present future directions. 


\section{System Model AND PRoblem Formulation}

We consider $N$ nodes equipped with half-duplex transceivers (i.e., nodes cannot transmit or receive packets simultaneously), where each node possess a unique $I D$ in the network. If every node is one hop away from every other node in the network, the network is a clique. The network is assumed to operate in a synchronous mode. Time is divided into discrete time slots, and each time slot is of duration $\tau$. Each time slot allows for a node to transmit or to receive one single packet (we do not allow multiple packet transmissions per time slot) in any of the $k$ available orthogonal channels.

We say that a collision occurs, when more than one node decide to transmit on the same channel simultaneously. We say a direct discovery occurs when at any given time slot only one node among many that might have selected the same channel $k_{i}$ transmits, while the remaining nodes are on the listening mode. We say an epidemic discovery or indirect discovery occurs, when it leads to the discovery of one or more neighbors through other neighbors, i.e., say $p$ receives a packet from $q$ and $q$ has already discovered some neighbors earlier (directly or indirectly) that $p$ has not discovered yet. Then, based on the epidemic approach followed in this work, $q$ disseminates all the IDs of the neighboring nodes already discovered via the transmitted packet.

Let $T$ be a random number denoting the time slots elapsed before any node discovered all its neighbors. Our work in this paper aims to answer the following questions. How large is $T$ ? How much is $T$ reduced using multichannel epidemic discovery comparatively with a single channel model? What is the impact of multichannel communications? How fast is epidemic discovery compared to the single channel model?

Towards this end, we develop two protocols: the first protocol is theoretical and serves as a benchmark for the evaluation of the second protocol, herein called the Multichannel Epidemic neighbor Discovery ALgorithm (MEDAL). Details about the two protocols are given below.

1) Epidemic multichannel neighbor discovery algorithm via coupon collection: this is a three phase multichannel neighbor discovery approach with epidemic dissemination. This model uses an idealistic scenario that is hard to implement in reality. However, it allows us to derive a bound, that serves as a lower bound for multichannel neighbor discovery time.

2) MEDAL-Multichannel Epidemic neighbor Discovery ALgorithm: in this protocol, we present a single phase multichannel neighbor discovery with epidemic dissemination that is evaluated both by simulations and experimentally.

\section{Multichannel Neighbor Discovery Algorithm}

\section{A. Single channel neighbor discovery}

Consider a clique of size $N$ nodes, as in a single-hop Slotted-Aloha network where any node can directly communicate with any other node. Under Slotted-Aloha, the probability that a node successfully transmits in any time slot is given by the probability a node transmits $p_{t}$, given that the remaining $N-1$ nodes remain silent. This probability, denoted by $p_{s}$, is given by:

$$
p_{s}=p_{t}\left(1-p_{t}\right)^{N-1}
$$

Differentiating $p_{s}$ with respect to $p_{t}$, the optimal transmission probability $p^{*}$ that maximizes the successful probability $p_{s}$ is given by $p^{*}=\frac{1}{N}$. Substituting $p^{*}$ into (1), we have:

$$
p_{s}=\frac{1}{N}\left(1-\frac{1}{N}\right)^{N-1} \approx \frac{1}{N e} .
$$

Neighbor discovery has been modeled by Towsley et al. [2] as a coupon collector problem, in which a collector (a wireless node in our case) wants to possess the whole set of $N$ different coupons (neighbor's presence in the network). Based on the coupon collector analysis, the average number of steps $T_{n}$ needed to discover all neighbors in a clique is given by

$$
E\left[T_{n}\right]=N H_{n} .
$$

where $H_{n}$ is the $n^{\text {th }}$ harmonic number $\left(H_{n}=\ln N+\theta(1)\right)$.

B. Multichannel epidemic neighbor discovery algorithm via coupon collection

Here, we consider a multichannel neighbor discovery approach that is implemented in three phases (each phase will be described in the sequel). We assume that all nodes know when the first two phases are finished; the next phase starts thereafter. While this approach is analytical, it serves as a benchmark for the evaluation of our realistic algorithms proposed herein.

Phase 1 - Multichannel coupon collector. Let $\mathcal{C}$, be the set of $k$ channels used for neighbor discovery, and $\mathcal{C}=$ $\left\{C_{1}, C_{2}, \ldots, C_{k}\right\}$. We first divide the nodes equally to the channels in order to minimize the number of nodes in each channel and hence reduce the number of collisions that affect the expected time for neighbor discovery. Since we assume that nodes have unique IDs, for example a modulo operation of the node ID by $k$ would be enough to accomplish it.

The idea of the first phase is that each of the nodes performs neighbor discovery of a smaller set of neighbors on the selected channel. Note that during this phase, epidemic dissemination is not assumed since this is equivalent to the single-channel neighbor discovery where it is assumed that a successful broadcasted packet is received by all nodes in the channel. An epidemic approach would be of interest to consider in case we have packet losses. Upon completion of neighbor discovery, in all channels the node with, say, the smallest ID number from each channel is selected as the leader to join the second phase (Phase 2). Otherwise, if the node ID is not offered for setting a leader of the group of nodes in the channel, and in order to keep the option for each of the nodes to be the leader, each node can choose randomly a number at the beginning of the first phase and disseminate this value among with its ID and the largest will become the leader, as in max-consensus algorithms (see, for example, [11]). 
Hence, using the coupon collector approach [2], the expected time $\mathbb{E}\left[T_{1}\right]$ for the first phase to terminate and each node to have discovered all the other nodes in the same channel is given by

$$
\mathbb{E}\left[T_{1}\right]=e\left\lceil\frac{N}{k}\right\rceil\left(\ln \left\lceil\frac{N}{k}\right\rceil+O(1)\right),
$$

where $\lceil\cdot\rceil$ is the minimum integer that is greater than or equal to its argument.

Phase 2 - Epidemic dissemination. In the second phase, all the $k$ selected nodes (leaders) from each channel move to a pre-specified channel in $\mathcal{C}$ and perform neighbor discovery in a single-channel. In this phase, epidemic dissemination of information plays a key role, since each node conveys information for all the nodes belonging in the same channel. Hence, any successful transmission by a node from any of the $k$ channels, leads to the discovery of the nodes of that channel by the remaining $k-1$ nodes in a single transmission (since each transmitted packet embeds the IDs of its neighbors in the channel they shared in Phase 1.

Again, using the coupon collector approach [2], the expected time $\mathbb{E}\left[T_{2}\right]$ for the first phase to terminate and each node to have discovered all the other nodes in the same channel is given by

$$
\mathbb{E}\left[T_{2}\right]=k e(\ln k+O(1)) .
$$

Phase 3 - Final broadcast. In the last phase, each of the leaders returns back to the initial channel and broadcasts its entries to the rest of the nodes in that channel. Thus, by the end of Phase 3, all nodes have discovered all the nodes in their neighborhood. Note that this phase requires only a single step.

As a result, by the end of the three phases, the total expected time $\mathbb{E}[T]$ is given by

$$
\begin{aligned}
\mathbb{E}[T] & =\mathbb{E}\left[T_{1}\right]+\mathbb{E}\left[T_{2}\right]+1 \\
& =e\left\lceil\frac{N}{k}\right\rceil\left(\ln \left\lceil\frac{N}{k}\right\rceil+O(1)\right)+k e(\ln k+O(1))+1 .
\end{aligned}
$$

This scheme fully exploits the existence of multiple channels and the epidemic dissemination of information. Note that in the case of one channel this is reduced to the single channel neighbor discovery. In practical scenarios, however, it is difficult to understand when the neighbor discovery of the first two phases terminate, and subsequently orchestrate the switching between the three phases without centralized information. Nevertheless, the expected time of this scheme is used as a benchmark for evaluating the performance of suggested algorithms in this paper.

\section{1) Terminating condition:}

Definition 1. [12] Let $T$ be the number of nodes discovered before obtaining one of each of $n$ types of nodes. Then, for any positive constant $c$

$$
\lim _{n \rightarrow \infty} P[T>n \mathrm{e}(\ln n+c)]=1-\mathrm{e}^{-\mathrm{e}^{-c}} .
$$

This Equation (6) states that, for large $n$, the number of trials should be very close to ne $\ln n$. This is an example of a sharp

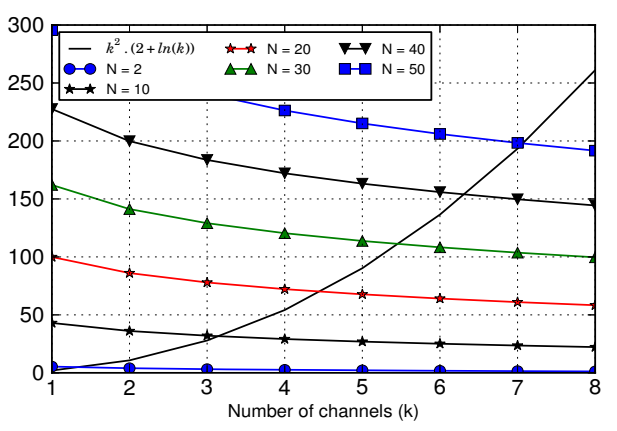

Fig. 1. Optimal number of channels given the average number of neighbors $k=\{1, \ldots ., 8\}$

threshold, where the random variable is closely concentrated around its mean.

The coupon collection problem is of the same type as ballsinto-bins problem [12]. Considering a Poisson approximation, we first assume that the number of balls in each bin is a Poisson random variable with mean $\ln n+c$, so that the expected total number of balls is $T=n e(\ln n+c)$. The probability that a specific bin is empty is then $e^{-(\ln n+c)}=\frac{e^{-c}}{n}$. Since all bins are independent under the Poisson approximation, the probability that no bin is empty (all nodes are discovered) is $\left(1-\frac{e^{-c}}{n}\right)^{n} \approx e^{-e^{-c}} \approx 1$.

2) Optimal number of channels: To obtain the optimal number of channels of our model, we differentiate $\mathbb{E}[T]$ with respect to $k$ (the number of channels), i.e.,

$$
\frac{d E(T)}{d k}=-\frac{N}{k^{2}}\left(\ln \frac{N}{k}+2\right)+\ln k+2 .
$$

Equating (7) to zero, we obtain to expression that we compare to obtain the optimal number of channels. We solve the resulting equation $N\left(\ln \frac{N}{k}+2\right)=k^{2}(\ln k+2)$ numerically. In Figure 1, the $y$-axis represents the term $N\left(\ln \frac{N}{k}+2\right)$ for different values of $N$ and $k$, while the $x$-axis represents the number of channels. The half parabola curve is the term $k^{2}(\ln k+2)$.To obtain the optimal $k$, we intercept the curves $N\left(\ln \frac{N}{k}+2\right)$ and $k^{2}(\ln k+2)$ obtained for different values of $k$ (for fixed $N$ ). Since $k$ must be an integer, we approximate $k$ to be the minimum next integer number larger than $k$ obtained by the interception of these curves. For example, for a clique of 30 nodes, we obtain numerically that $k=5.5$. Hence, the number of channels minimizing the expected time for a node to discover all its neighbors is 6 channels.

\section{MEDAL - MUlTiCHANNEL EPIDEMiC NeIGHBor DISCOVERY ALGORITHM}

In this section, we propose an single phase algorithm. It can be easily shown that the multichannel epidemic discovery algorithm converges in $\tau, \tau<\infty$ steps with probability 1 . The behavior of the algorithm can be modeled as an absorbing Markov chain. However, because of the transition probabilities are very different on each link, it is not possible to extract a closed form solution. In addition, the number of possible states 
grows exponentially with the number of nodes $N$, while the number of channels $k$ affect the transition probabilities only.

We assume that each node is synchronized, and the time is divided into time slots of duration $\tau$. We also assume that every node on the system knows the number of channels to use beforehand. A node also keeps an array (network table) that will hold the IDs of all its neighbors.

Description: We describe MEDAL briefly in the algorithm 1. (i) in each time slot each node selects a channel $k_{i}$ uniformly at random, (ii) if the adaptive mode is used (unknown $N$ ) each node recomputes the transmission probability $p_{t}$, (iii) each node attempts to transmit with probability $p_{t}$. If a transmission occurred, it includes the list of neighbors that it has discovered so far (epidemic dissemination). (iv) Otherwise, if a packet was received, it adds new neighbors, or it updates its list of neighbors.

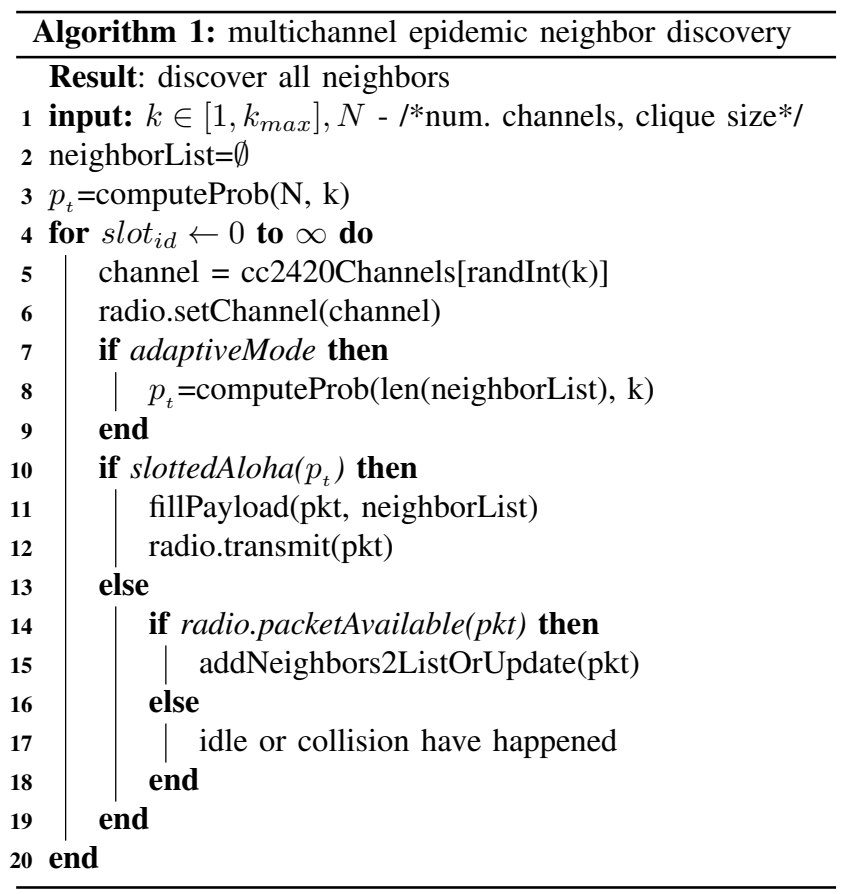

\section{A. Optimal transmission probability}

Let $i$ and $j$ be two random nodes that are synchronized to the same channel at time slot $t$. Let $p_{s}$ be the probability that node $i$ discovers node $j$ in a given time slot in any given channel. Node $i$ discovers node $j$, if (a) node $j$ transmits with probability $p,(b)$ none of the remaining $N-1$ nodes in the channel transmit. Under this conditions, node $i$ successfully receives a packet from node $j$ with probability $p_{s}$, given by

$$
p_{s}=\frac{1}{k} p\left(1-\frac{p}{k}\right)^{N-2}(1-p) \text {. }
$$

The differentiation of (8) with respect to $p$ gives the optimal transmission probability $\left(p^{*}\right)$.

$$
p^{*}=\frac{2 k+N-1-\sqrt{(2 k+N-1)^{2}-4 k N}}{2 N} .
$$

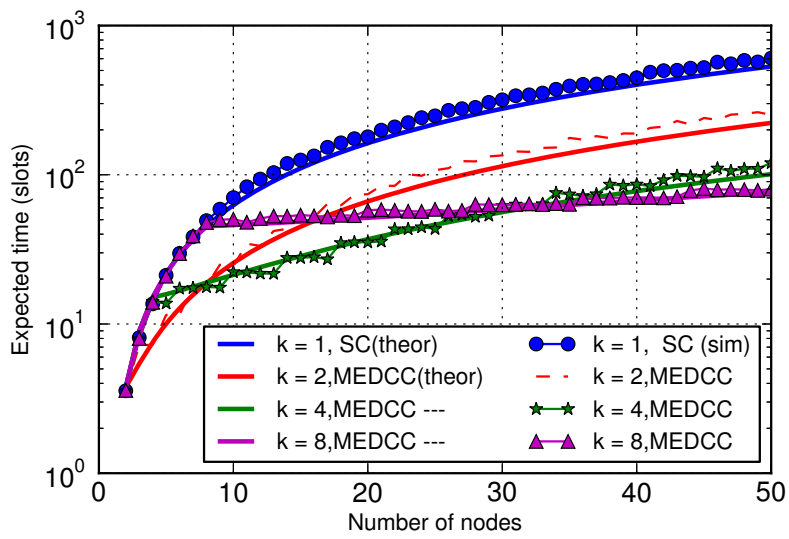

Fig. 2. Comparison between analytical and simulation results of multichannel epidemic neighbor discovery via coupon collection (MEDCC) and single channel (SC) for $C=\{1,2,4,8\}$.

We use $p^{*}$ in both simulations and experimental evaluations.

\section{Performance Evaluation}

We evaluate the different neighbor discovery schemes both by simulations and experimentally. Unless otherwise stated, we will consider a single clique scenario, varying the number of nodes from 2 to 50 , and the number of channels from 1 to 8 . Each point in the figures is obtained by averaging the result of 300 simulations. The results at each data point are within $95 \%$ confidence interval. Experimentally, we implemented MEDAL in $\mathrm{C}$ using Telos $\mathrm{B}$ motes, running the Contiki [13] operating system.

\section{A. Multichannel epidemic neighbor discovery}

Figure 2 shows the total discovery times for the multichannel neighbor discovery algorithm as a function on network side. Results show both the average discovery times (obtained by Monte Carlo simulations) and the expected discovery times. Each curve represents different number of channels, from 1 (in which case we recover the single-channel neighbor discovery algorithm introduced in [2]) to 8 . The figure shows that the multi-channel algorithm achieves uniformly better discovery times than the single channel version (irrespectively of the clique size and the number of channel used). The improvements increase when the clique size increases. We can also observe that the optimal number of channels to use for neighbor discovery depends on the clique size. Finally, we also see that the analytical results agree well with the results obtained by simulations.

\section{B. Simulation and experimental results for MEDAL}

Figure 3 shows the average neighbor discovery times obtained by the MEDAL algorithm as a function of the clique size, and for different number of channels. For reference, the results for the single-channel neighbor discovery protocol from [2] is also included. For both schemes, we have used the theoretically optimal transmit probability.

The simulations and experimental (dashed lines) results demonstrate that MEDAL obtains similar benefits as the less 


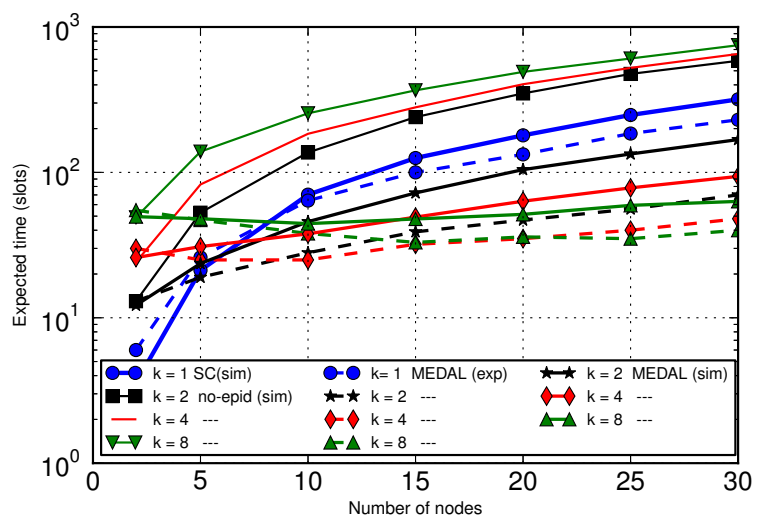

Fig. 3. Comparison between multichannel discovery, multichannel epidemic discovery, and direct discovery (single channel). The results show that multichannel epidemic neighbor discovery is better for medium size and larger networks, while single channel neighbor discovery is better for small networks $(N \leq 5)$. The results also show that multichannel neighbor discovery used alone is a bad option.

practical multi-channel coupon collection algorithm. For a large clique size of $N=30$, the single-channel protocol requires over 200 time slots to discover all neighbors, while MEDAL needs only 150 time slots in the 2-channel operation, and 55 time slots in the 8-channel operation.

1) Impact of clique size: However, MEDAL is not uniformly better than the single-channel algorithm. For small network sizes, the positive effect of reduced contention is not able to offset the fact that nodes meet more infrequently on the same channel. In particular, there are no benefit of MEDAL when the clique size is less than 5 nodes, and the number of channels used by MEDAL should always be smaller than the number of nodes.

2) Impact of epidemic discovery: Finally, to highlight the importance of the epidemic information dissemination component, we simulate MEDAL without this feature. As expected, this results in significantly increased neighbor discovery times, see Figure 3. In fact, without epidemic information dissemination, the discovery times will be worse than that of the single-channel protocol, and the performance degrades with the number of channels used.

3) Speed of convergence: To illustrate the convergence properties of the MEDAL algorithm, Figure 4 shows the average fraction of nodes discovered as function of time. The results are shown for a clique size of 30 nodes, allowing MEDAL to use 8 channels for neighbor discovery. We note that MEDAL converges quickly and improves the total discovery time with a factor of 6 over the single-channel algorithm.

\section{CONCLUSIONS}

In this paper, we first presented a protocol for neighbor discovery in multichannel networks with epidemic information dissemination as a coupon collector problem, that is implemented in three phases. It provides a good benchmark for evaluating the performance of proposed approaches. Secondly, we presented MEDAL, a distributed randomized algorithm for multichannel epidemic neighbor discovery. We demonstrate

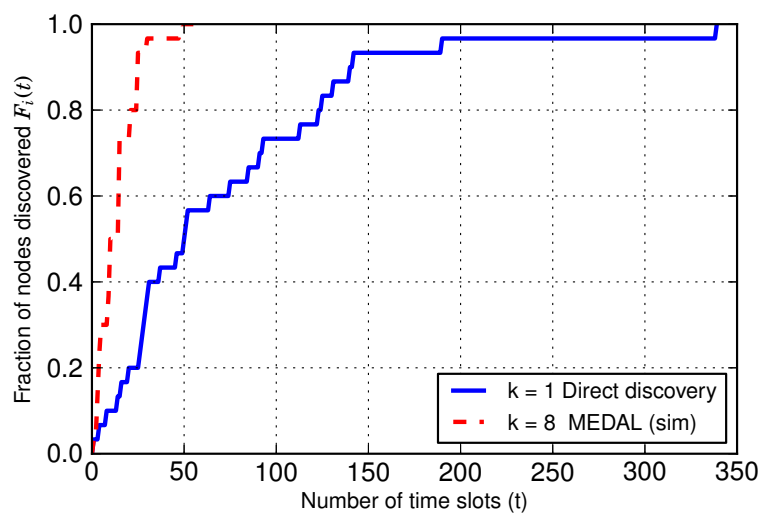

Fig. 4. Comparison between multichannel epidemic discovery and single channel neighbor discovery for a network size of $N=30$ nodes.

that MEDAL performs much better than the single channel neighbor discovery, especially in dense networks, but it could be a bad choice for very small networks (i.e., $N \leq 5$ ). Finally, we experimentally evaluated MEDAL on a real testbed where the simulation results have been validated.

Future directions include:

(a) extend the model to consider the case where the network size is unknown;

(b) include loss probability on the communication links between the nodes into the model;

(c) consider networks other than the clique.

\section{REFERENCES}

[1] P. Kyasanur and N. H. Vaidya, "Capacity of multi-channel wireless networks: impact of number of channels and interfaces," pp. 43-57, 2005.

[2] S. Vasudevan, D. Towsley, and D. Groeckel, "Neighbor discovery in wireless networks and the coupon collector's problem," In Proc. of ACM MobiCom, pp. 181-192, 2009.

[3] A. Keshavarzian, E. Uysal-Biyikoglu, F. Herrmann, and A. Manjeshwar, "Energy-efficient link assessment in wireless sensor networks," In Proc. of IEEE INFOCOM, pp. 1751-1761, 2004.

[4] K. Pister and L. Doherty, "Tsmp: Time synchronized mesh protocol," IASTED Distributed Sensor Networks, pp. 391-398, 2008.

[5] T. Watteyne, A. Mehta, and K. Pister, "Reliability Through Frequency Diversity: Why Channel Hopping Makes Sense," PE-WASUN, 2009.

[6] P. T. Eugster, R. Guerraoui, A.-M. Kermarrec, and L. Massoulié, "Epidemic information dissemination in distributed systems," Computer, vol. 37, no. 5, pp. 60-67, 2004.

[7] P. T. Eugster, R. Guerraoui, S. B. Handurukande, P. Kouznetsov, and A.M. Kermarrec, "Lightweight probabilistic broadcast," ACM Transactions on Computer Systems (TOCS), vol. 21, no. 4, pp. 341-374, 2003.

[8] M. Chrobak, L. Gasieniec, and W. Rytter, "A randomized algorithm for gossiping in radio networks," Computing and Combinatorics, pp. 483492, 2001.

[9] N. Karowski, A. C. Viana, and A. Wolisz, "Optimized asynchronous multi-channel neighbor discovery," pp. 536-540, 2011.

[10] N. Mittal, Y. Zeng, S. Venkatesan, and R. Chandrasekaran, "Randomized distributed algorithms for neighbor discovery in multi-hop multi-channel heterogeneous wireless networks," pp. 57-66, 2011.

[11] F. Iutzeler, P. Ciblat, and J. Jakubowicz, "Analysis of max-consensus algorithms in wireless channels," IEEE Transactions on Signal Processing, vol. 60, no. 11, pp. 6103-6107, Nov. 2012.

[12] M. Mitzenmacher and E. Upfal, Probability and Computing: Randomized Algorithms and Probabilistic Analysis. Cambridge University Press, April 2005.

[13] A. Dunkels, B. Gronvall, and T. Voigt, "Contiki-a lightweight and flexible operating system for tiny networked sensors," pp. 455-462, 2004. 